Int. J. Dev. Biol. 52: 993-998 (2008)

doi: $10.1387 / \mathrm{ijdb} .082582 \mathrm{pc}$

\title{
Identification and gene expression of versican during early development of Xenopus
}

\author{
PAOLA CASINI 1,\#, MICHELA ORI*,1,\#, ANGELA AVENOSO ${ }^{2}$, ANGELA D'ASCOLA $^{2}$, PAOLA TRAINA ${ }^{2}$, \\ WALTER MATTINA ${ }^{2}$, ROBERTO PERRIS ${ }^{3}$, GIUSEPPE M. CAMPO ${ }^{2}$, ALBERTO CALATRONI ${ }^{2}$, IRMA NARDI ${ }^{1}$ \\ and SALVATORE CAMPO ${ }^{2}$ \\ ${ }^{1}$ Unit of Cellular and Developmental Biology, Dept. of Biology, University of Pisa, Pisa, ${ }^{2}$ Dept. of Biochemical, Physiological and \\ Nutritional Sciences, School of Medicine, University of Messina, Policlinico Universitario, Torre Biologica, Messina and \\ ${ }^{3}$ Lab. of Tumour Biology and Stem Cells, Dept. of Genetics, Microbiology and Anthropology, University of Parma, Parma, Italy.
}

\begin{abstract}
The chondroitin sulfate proteoglycan (PG) PG-M/versican is known to be a primary component of the vertebrate embryonic extracellular matrix and, in the mouse, functional abrogation of the versican gene leads to severe cardiovascular malformations and embryonic lethality. In order to provide a means for approaching the study of the role of versican during embryogenesis, we have cloned the Xenopus versican cDNA and we have performed in situ hybridization on embryos at different stages of development. We showed maternal Xversican transcription, as well as a previously undocumented early expression of the PG during gastrulation and neurulation. At later stages of development, spatial transcription of $X v$ versican correlates with the patterns of migrating neural crest cells (NCC) and it is expressed in embryonic regions representing the final sites of arrest of NCC. Xversican mRNA was also detected in a subpopulation of trunk NCC migrating into the fin, in tissues flanking the trunk NCC ventral migratory pathway and in post-migratory cranial skeletogenic NCC. Further embryonic sites expressing Xversican were the pronephros, pronephric ducts, heart anlage and branchial pouches. These findings constitute the basis for future studies aimed at clarifying unresolved aspects of versican function during embryogenesis.
\end{abstract}

KEY WORDS: chondroitin sulfate proteoglycan, versican, embryo, neural crest, Xenopus laevis

Versican is a chondroitin sulfate proteoglycan (PG) belonging to the hyalectan subgroup of PGs including aggrecan, neurocan, brevican and CD44 and having in common an N-terminal G1 domain embodying the binding site for hyaluronan. Aggrecan, neurocan and brevican show a rather restricted tissue distribution (e.g. aggrecan is most prominent in cartilage, whereas neurocan and brevican are predominantly expressed in the central nervous system), whereas versican is present in a variety of tissues and organs, implying that it may exert a diverse biological function (lozzo, 2005). In addition to the hyaluronan-binding domain, hyalectans show common structural features such as a set of epidermal growth factor (EGF), lectin and complement regulatory protein (CRP) in the carboxy-terminal portion (G3 domain) of the molecule (Zimmermann and Ruoslahti, 1989; Shinomura et al., 1993). The glycosaminoglycan (GAG) attachment regions are located in the middle portion of the protein and are different in the four extracellular matrix (ECM) PGs of the hyalectan subgroup. In versican, two different GAG attachment domains have thus far been identified in higher vertebrates and denoted GAG $\alpha$ and GAG $\beta$. Both domains (exons) are present within the parental V0 isoform while they are differentially spliced out in isoforms $\mathrm{V} 1$ and V2 (Zimmermann and Ruoslahti, 1989; Shinomura et al., 1993).

The complex structure of versican provides the basis for its multiple and sometime contrasting effects on cellular functions, such as cell adhesion, cell migration, cell proliferation and apoptosis, as well as on morphogenesis. For instance, in the nervous system, versican seems to mainly act as barrier molecule affect-

Abbreviations used in this paper: CRP, complement regulatory protein; ECM, extracellular matrix; EGF, epidermal growth factor; GAG, glycosaminoglycan; NCC, neural crest cell; PG, proteoglycan.

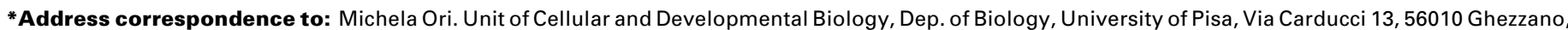
Pisa, Italy. ; Fax: +390502211495. e-mail address: mori@biologia.unipi.it
}

\# Note: Both authors contributed equally to this work.

Published online: 20th June 2008

$0214-6282 / 2008 / \$ 35.00$

(C) UBC Press

Printed in Spain 


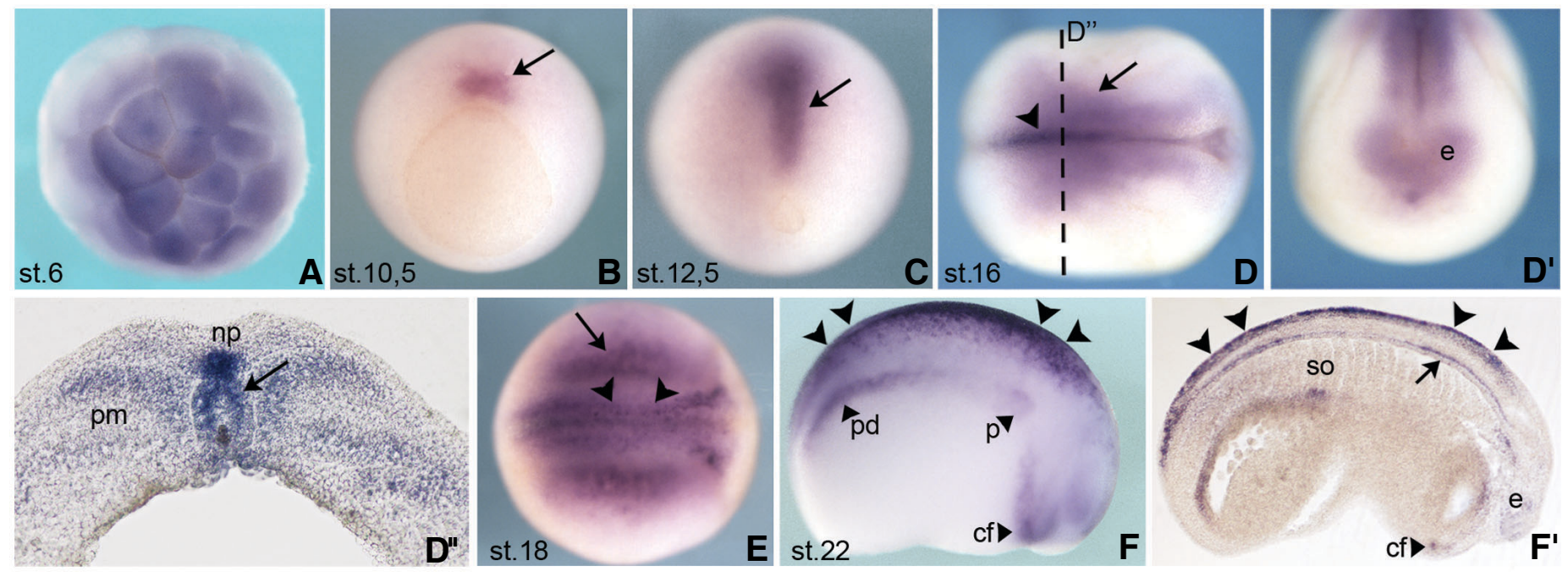

Fig. 1. Spatial expression of Xversican mRNA in early Xenopus laevis embryos. (A) Xversican maternal transcript is distributed in the animal pole blastomeres of the blastula stage embryo (st. 6). (B,C) During gastrulation (st. 10.5 and 12.5 respectively) the mRNA is localized in the gastrulating dorsal axial mesoderm (arrows). (D) Dorsal view of a midneurula stage embryo (st. 16): the expression can be detected in the paraxial mesoderm (arrow) and in the midline of neural plate (arrowhead). (D') Frontal view of the embryo shown in (D) exhibiting Xversican transcript in the eye field (e). (D') Vibratome transverse section of the embryo in (D) revealing the presence of Xversican mRNA in the notochord (arrow), in the medial ventral domain of the neural plate (np) and in the paraxial mesoderm (pm). (E) Dorsal view of late neurula stage embryo (st. 18). Xversican is detectable in the premigratory trunk NCC (arrowheads) and in the lateral plate mesoderm (arrow). (F, $\mathbf{F}^{\prime}$ ) At stage 22, Xversican transcript is still present in the trunk NCC (arrowheads) and appears in the cardiac field (cf), pronephrus (p) and pronephric ducts (pd). (F') Vibratome sagittal section reveals the labelling in the floor plate of the neural tube (arrow) and the down regulation of Xversican in the forming somites (so).

ing axon growth, cell migration and plasticity and these functions appear to be performed through its GAG chains (Schmalfeldt et al., 2000; Bradbury et al., 2002; Wu et al., 2005; Cattaruzza and Perris, 2006).

A first evidence of a crucial role of versican during embryogenesis derives from the finding that the heart defect (hdf) mouse is a recessive lethal one that has arose from a transgene insertional mutation in the versican gene causing its almost complete abrogation. Embryos homozygous for the transgene die in utero by embryonic day 10.5 and exhibit specific defects along the cardiac axis (Mjaatvedt et al., 1998). Intringuingly, this phenotype very closely resembles that exhibited by the hyaluronan synthase 2 (Has2) knock-out mouse (Camenisch et al., 2000) and, accordingly, has thus far not allowed to fully dissect the functional role of the PG during development. However, previous studies have underscored a crucial role of isoforms V0 and V1 during trunk neural crest cell (NCC) migration suggesting a chemorepulsive function (Landolt et al., 1995; Dutt et al., 2006). Other investigations propose instead that versican is a component of the ECM of NCC pathways controlling directionality of cell movement through a mechanism of "inverse haptotaxis" (Perris, 1997; Perissinotto et al., 2000; Perris and Perissionotto, 2000), consistent with previous findings in the white mutant axolotl embryo harbouring a genetic deficiency in versican expression (Stigson et al., 1997; Perris and Perissinotto, 2000). Because the importance of versican during development, we decided to address its role by using Xenopus laevis embryo as model system.

We have previously determined the expression pattern of the Xenopus Has genes (Nardini et al., 2004) and we have shown that Has2 is widely expressed during development and is implicated in somitogenesis and precursor cell migration (Ori et al., 2006).Since hyaluronan is one of the main versican binding partners we asked whether the versican gene expression is spatiotemporally coordinated with that of Has2.

In the present work we cloned the full length Xenopus versicancDNA (V0) and analyzed its expression pattern during embryogenesis.

\section{Results}

\section{Xversican cloning}

In the present study the full-length versican cDNA (V0) was identified in Xenopus laevis embryos (st. 30) as a sequence of $11.559 \mathrm{bp}$, codifying a putative core protein of 3852 amino acids. The comparison of the aminoacid sequence with that of other vertebrate orthologues shows the typical structure of the versican core protein composed of a G1 hyaluronan-binding domain, flanked by the two well-described GAG attachment regions, $\mathrm{GAG} \alpha$ and $\mathrm{GAG} \beta$, and the multimodular $\mathrm{G} 3$ domain embodying an EGF repeat, lectin-like module and a CRP repeat. Whereas the $\mathrm{G} 1$ and $\mathrm{G} 3$ domains of Xversican show high homology (from $71 \%$ in the hyaluronan-binding $\mathrm{G} 1$ domain to $92 \%$ in the lectin-like region) with the human versican the homology drastically decreases in the GAG $\alpha$ and GAG $\beta$ domains (19.5\% compared with the human versican aminoacid sequence). Moreover, the Xversican GAG $\alpha$ and GAG $\beta$ domains appear $16 \%$ longer than the corresponding human regions. In addition to the full length $V 0$ isoform we identified spliced variants corresponding to the isoforms V1, V2 and V3 (the corresponding nucleotide sequences have been submitted to GenBank with the following accession numbers: V0: 
EF120478, V1: EU204646, V2: EU204648, V3: EU204650).

\section{Xversican mRNA expression during embryogenesis}

In order to detail the Xversicanspatio-temporal gene expression during development we performed in situ hybridization experiments using an antisense RNA digoxigenin labelled probe designed to bind the G3 domain of the Xversican mRNA.

Xversican transcripts were first detected at blastula stage (st. 6) in the animal pole blastomeres suggesting that it corresponds to a maternal transcript (Fig. 1A). Zygotic Xversican expression was then detected at early gastrula stage (st. 10.5) in the presumptive axial mesoderm fated to form the notochord during gastrulation (st. 12.5; Fig. 1B, C). Starting from midneurula Xversican expression becomes clearly detectable in the paraxial mesoderm and anteriorly in the eye field (Fig. 1D, D'). Coronal sections of the same stage embryos revealed $X$ versican expression in the notochord and in the medio-ventral domain of the neural plate (Fig. 1D"). At late neurula stage (st. 18) labelling was detectable in premigratory cells of the trunk NCC and in the lateral, non-segmented plate mesoderm (Fig. $1 \mathrm{E})$. At stages 22, Xversican expression appeared in the cardiac field, in the pronephros and in two symmetrical lateral strips, corresponding to the pronephric ducts (Fig. 1F). Sagittal sections of the same stage embryos revealed a down-regulation of Xversican mRNA in the forming somites, while a signal was still detectable in the floor plate of the neural tube (Fig. 1F').

\section{Xversican expression in the branchial arches}

Starting from tailbud stages (st. 26), Xversican mRNA was observed in the branchial arches (Fig. 2A, A', A"). Horizontal sections show that, at this stage, Xversican expression is restricted to the endodermal component of the branchial pouches (Fig. 2A"). At stage 37, after the completion of NCC migration, Xversican mRNA is expressed by the cranial neural crest component of the branchial arches, being organized in a pattern reminiscent to that of $X$ Sox 9 , a specific skeletogenic cranial NCC marker (Fig. 2B, B', B'). This pattern of Xversican expression is very similar to that one previously observed for XHas2 (Nardini et al., 2004). Accordingly, in later stages of larval development (st. 41), Xversicanis expressed in chondrogenic precursor cells forming the visceral skeletal elements, as confirmed by comparing Xversican expression with that of XSox9 (Fig. 2C, C', C'). On the whole, Xversican transcript expression in the branchial arches overlaps that of XHas2, which appears first in the endodermal component of the pouches and subsequently in the cranial NCC chondrogenetic precursors (unpublished results), while both genes are absent in migrating cranial NCC (cf. Nardini et al., 2004).

\section{Xversican is produced by a subset of trunk NCC}

At tailbud stage Xversican expression is detectable in a subpopulation of cells migrating into the fin (Fig. 3). From stage 25-26 onwards Xenopus trunk NCC initiate their migration along two major pathways: a dorsal and a ventral pathway (Krotoski et al., 1988; Collazo et al., 1993, see Fig. 3A). Dorsally migrating ones reach the dorsal fin and then migrate around the tail tip and along the ventral fin (Fig. 3B, C). It has been suggested that these cells might contribute to the dorsal and ventral fin structures (Collazo et al., 1993). As Xversican is present in trunk premigratory NCC we would like to suggest that dispersed Xversican positive cells within the dorsal and ventral fin may represent dorsally migrating NCC. The ventral migratory pathway is divided in two different routes, the majority of NCC migrate between the neural tube, somites and notochord, while a minor lateral pathway is formed by NCC moving underneath the ectoderm lateral to the neural tube (cf. Fig. 3A). These populations of migrating trunk NCC do not appear to transcribe Xversican.

It is interesting to note that premigratory trunk NCC express Xversican (from st. 19) as well as XHas2 mRNA (Nardini et al., 2004), whereas from stage 26 onwards Xversican seems to be only synthesized by a subpopulation of trunk NCC migrating along the dorsal pathway (Fig. 3B, C, D). It remains to be clarified whether Xversican gene expression may identify a premigratory subset of NCC that are destined to specifically migrate along the dorsal pathway, or whether all precursors cells are initially able to produce versican but only a subpopu-

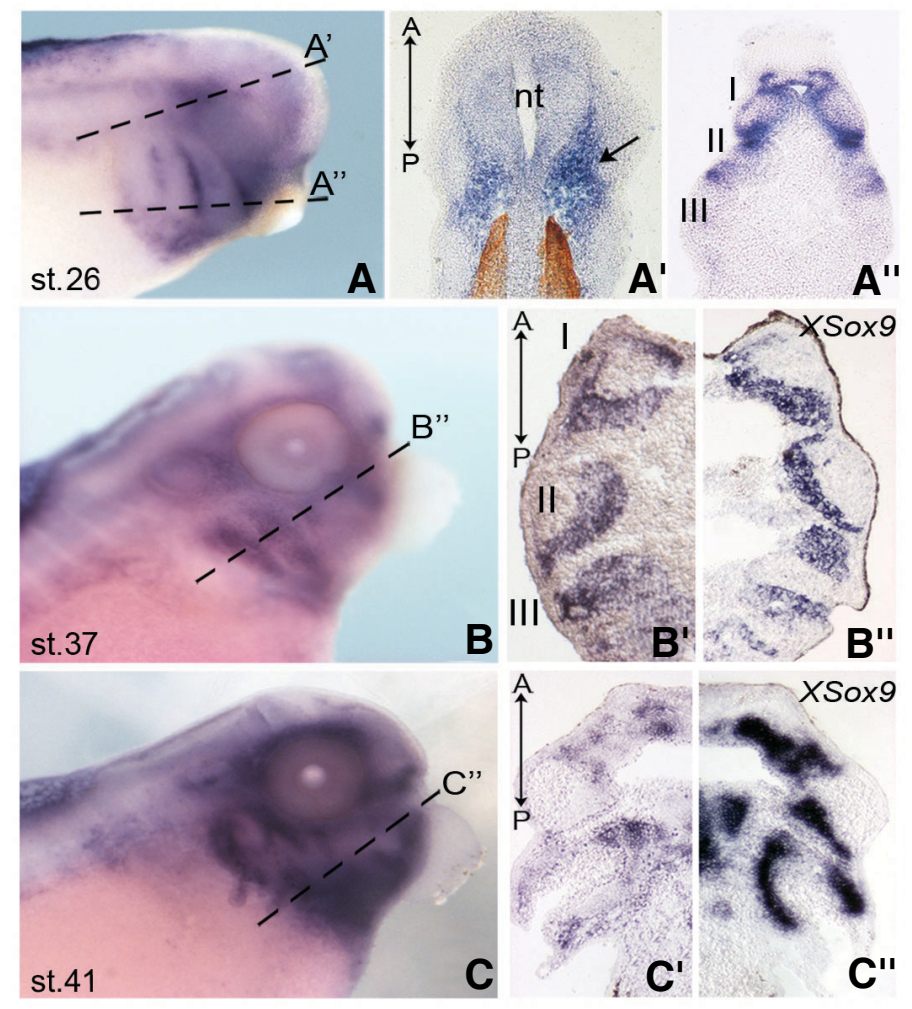

Fig. 2. Spatial distribution of Xversican mRNA during branchial arches development. In each panel dashed lines indicate the level of the sections. (A) Lateral view of a tailbud stage embryo (st. 26) showing Xversican expression in the head region. (A') Horizontal vibratome section highlighting Xversican transcript in the cranial mesenchyme (arrow). In brown, the immunolabelling for 12/101 antibody shows the head somites. ( $\left.\mathbf{A}^{\prime \prime}\right)$ Horizontal vibratome section showing Xversican expression in the endoderm of the pouches. (B, $\left.\mathbf{B}^{\prime}, \mathbf{B}^{\prime \prime}\right)$ At stage 37 Xversican mRNA is expressed by the cranial NCC migrated into the branchial arches ( $B^{\prime}$, horizontal section) as confirmed by the expression of the NCC marker XSox9 in (B). (C, $\mathbf{C}^{\prime}, \mathbf{C}^{\prime \prime}$ ) Later in development (st. 41) Xversican transcript remains expressed in chondrogenic precursor cells as shown in the horizontal section in $\left(C^{\prime}\right)$ compared to the expression of XSox9 in (C). In each panel roman numbers indicate respectively the first, second and third/fourth branchial arches. nt, neural tube. 


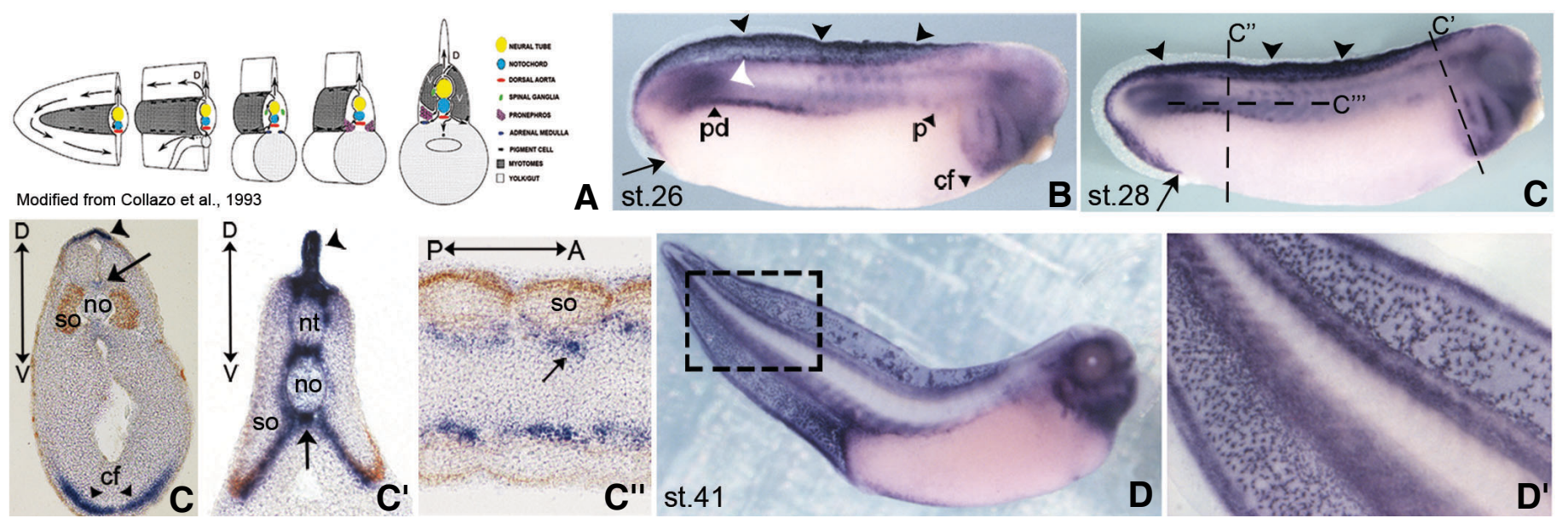

Fig. 3. Expression of Xversican mRNA in trunk neural crest cells and mesodermal derivatives in Xenopus embryos. Dashed lines indicate the level of the sections. (A) Diagram of the Xenopus trunk neural crest migration pathways, modified from Collazo et al., 1993. (B,C) Lateral views of stage 26 and 28 embryos respectively, showing Xversican transcript in trunk NCC migrating into the fin (compare arrows in (B,C), in the cardiac field (cf) (see also $C^{\prime}$ ), pronephrus (p) and pronephric ducts ( $\left.p d\right)$. Xversican mRNA is localized in the caudal presomitic mesoderm of the tailbud (white arrow) and in branchial arches (see Fig. 3). (C') Transverse vibratome section showing Xversican expression in the cardiac field (cf) and in the trunk NCC migrating, along the dorsal pathway, into the fin (arrowhead). A faint signal is still detectable in the ventral floor plate (arrow), while somites (so), labelled with the muscle cells specific marker 12/101 antibody, do not express Xversican. (C') Transverse vibratome section showing Xversican mRNA distribution at trunk level. NCC expressing Xversican are clearly detectable into the dorsal fin (arrowhead). Labelling signal appears in the ventro-medial portion of the somites (so), around the neural tube (nt) and the notochord (no) and in the ipochord (arrow), whereas no expression could be detected within the myotomes stained with the 12/101 specific antibody. (C'") Horizontal section showing somites stained with the 12/101 antibody and the metameric distribution of the Xversican transcript in the sclerotomic portion of the somites (arrow). (D, D') At stage 41 Xversican expressing cells dispersed into the dorsal and ventral fin are still visible. D, dorsal trunk NCC pathway; $V$, ventral trunk NCC pathway.

lation of trunk NCC maintains this capability during migration. By stage $41 X$ versicanexpression is still clearly distinguishable in cells dispersed through dorsal and ventral fins (Fig. 3D, D').

\section{Xversican expression in mesodermal derivatives}

From stage 26 onwards, Xversican transcript also appears in the presomitic mesoderm of the tailbud and in the heart anlage (Fig. 3B, C, C'). Along the body axis, Xversican mRNA shows a metameric distribution following a rostral-to-caudal sequence of somites (Fig. 3B). This pattern was analogous to that previously reported in the chick embryo (Perris et al., 1996; Landolt et al., 1995; Perissinotto et al., 2000). Serial transverse and horizontal sections of embryos previously subjected to whole-mount in situ hybridisation, were stained with the 12/101 antibody to reveal muscle cells, such as to allow to define the Xversican transcript expression pattern in this embryonic region in more in detail. At stage 26 only posterior somites appeared labelled in their ventromedial (sclerotomic) portion, whereas no expression could be detected in anterior somites (Fig. 3C). The hybridization signal was also present around the neural tube and the notochord, where sclerotome cells gradually spread (Fig. 3C', C', C'”). This expression pattern very close resembles the ventral migratory pathway and, similarly to what has been previously shown in chick embryo, XversicanmRNA may be transcribed by tissues flanking the NCC migratory pathways (Perissinotto et al., 2000).

\section{Discussion}

In this study we show that Xversican is early and dynamically expressed during Xenopus embryogenesis. Somewhat unexpectedly $X$ versican seems to be a maternal gene and the $P G$ is expressed early on in the axial mesoderm of the gastrulating embryo. Of particular interest is also to note the concomitant expression of Xversican with that of XHas2 in the presomitic mesoderm during neurulation, a stage in which the activity of the synthase is critical to prevent myoblasts from undergoing apoptosis (Ori et al., 2006). It is therefore intriguing to envision a synergic role of hyaluronan and versican in structuring the ECM surrounding myoblasts during this critical step of myogenesis. Notably, both XHas2and the Xversicangene are down-regulated as myogenesis proceeds during the antero-posterior segmentation of the presomitic mesoderm.

Later in development versican appears to represent a synthetic product of a subset of cells located in the dorsal and ventral fin that are likely trunk NCC dorsally migrating. For obvious reasons, this population has not a counterpart in chick or mouse embryos and probably represents a phylogenetically older trunk NCC subpopulation that in amphibia has the putative function of structuring the interstitial ECM of the fin or to give rise to presumptive secretory cells as suggested by Collazo and colleagues (1993). Trunk NCC migrating ventrally do not express Xversican, but it is likely that Xversican deposition in the ECM through which they migrate may be implicated in their guidance, as previously described in the chick and axolotl embryos. Identification of $X$ versican gene now opens the possibility to clarify in a different model the highly debated role (not readily resolvable in mammals) of versican in NCC migration (Perissinotto et al., 2000; Dutt et al., 2006), thanks to the possibility to more precisely manipulate its expression in the embryo by means of gene loss of function experiments.

Cranial NCC of the branchial arches do similarly not express $X$ versican during their migratory phase, but Xversican transcrip- 
tion occurs at their final site of arrest, i.e. in the pharyngeal pouches of the early tailbud embryo. Xversicanexpression is then detected in the NC-derived chondrogenic precursors giving rise to the skeletal components of the branchial arches. This pattern of expression of the PG largely overlaps with that of XHas2, suggesting that the functional relationship between hyaluronan and versican is initiated early on during embryonic development and may also be crucial for cranial NCC differentiation and craniofacial morphogenesis. Xversican and XHas2 exhibit overlapping expression also in the heart anlage, where these two ECM molecules seem to accomplish important functions as shown by the phenotypes exhibited both by Versican and Has2 null mice (Mjaatvedt et al., 1998; Camenisch et al., 2000) and by our previous observations following $X$ Has 2 abrogation in the early Xenopus embryo (Ori et al., 2006).

In addition to the branchial pouches, Xversican transcript is detectable early on in other embryonic sites that later in development are colonized by trunk NCC, such as the pronephros, the pronephric duct and the heart. It has previously been suggested that during chick NCC migration through the trunk region versican may act as an "inversed haptotactic factor", that is an ECM factor that guides movement from a lower to a higher concentration of the factor, causing arrest of the cells at sites of peak concentrations of it (Perissinotto et al., 2000; Perris and Perissinotto, 2000). Further functional studies in the Xenopus embryo are likely to clarify this mechanism still debated in literature.

$X$ versicantranscription seems also to accumulate in signalling zones, such as the floor plate and the dorsal half of the notochord, suggesting that the PG may participate in inductive events controlling CNS development. On the whole the results add to our understanding of versican gene expression during early vertebrate development and further suggest that it may represent an ancestral PG with multiple evolutionary conserved functions and important tissue specific roles waiting to be further unveiled.

\section{Materials and Methods}

\section{cDNA walking by 5'GENE RACE technology for full-lengh Xenopus} laevis versican $m R N A$ characterisation

We carried out the Xenopus laevis versican mRNA characterisation by the cDNA walking procedure and the 5'Gene Rapid Amplification of cDNA End method. Total RNA was extracted from stage 30 Xenopus laevis embryos using the Trizol reagent (Invitrogen, USA), according to manufacturer's instructions.

An EST clone containing a putative fragment of the Xenopus laevis versican gene has been initially identified by an in silico screening. The corresponding cDNA (clone XL078I14) was obtained from NIBB/NIG/ NBRP Xenopus laevis EST project (http://xenopus.nibb.ac.jp/). This clone was sequenced, aligned with human and chick versicansequences and used to design specific reverse oligos for the following 5'Gene Racer method (Fromhan, 1993). The cDNA walking was conducted by using the 5' GENE RACE commercial kit (Invitrogen, USA), according to the supplier's instructions, with modified forward primers to obtain highly specific PCR products. Each PCR product was cloned using the TOPO TA Cloning for sequencing kit (Bernard et al., 1994) and the TOP ${ }_{10}$ E. Coli competent cells (Invitrogen, USA), as suggested by the manufacturer. A 15 random selected clones were sequenced on both DNA strands, using the T3 and T7 internal oligos for sequencing and the ABI PRISM BigDye terminators cycle sequencing ready reaction kit (v. 1.1, Applied Biosystems, USA). The polynucleotides fragments obtained were separated by capillary electrophoresis on the ABI PRISM 310 Genetic Analyzer (Applied
Biosystems, USA) and the electropherograms were analysed by the free ware Finch TV software (Geospiza, UK). The cDNA walking was always performed designing three new reverse oligos in the 5' end of the each newly identified nucleotide sequence.

\section{In situ hybridization and immunohistochemistry}

$X$ versican digoxygenin labelled antisense probe was transcribed from a partial cDNA clone corresponding to the region codifying for the EGF/ Lectin/CRP domains in order to be able to detect all the Xversican splice variant mRNAs. Embryos were staged according to Nieuwkoop and Faber (1967). Whole-mount in situ hybridization was performed essentially as described by Harland (1991). BM purple has been used as alkaline phosphatase substrates. Alternatively, tissue sections were obtained from embryos fixed 2 hours in $4 \%$ paraformaldeyde in PBS and subsequently cryo-protected with $20 \%$ sucrose in PBS. The $14 \mu \mathrm{m}$ cryosections obtained were processed under stringent conditions $\left(65^{\circ} \mathrm{C}, 50 \%\right.$ formamide; washing solution $1 \times \mathrm{SSC}, 50 \%$ formamide at $65^{\circ} \mathrm{C}$ ) following the protocol reported in Strahle et al., (1994), with minor modifications. Control experiments were performed with sense probes (data not shown). The XSox9 antisense probe was prepared according to Spokony et al., (2002). For histological examination, whole-mount processed embryos were embedded in a gelatin albumin solution $(0.5 \%$ gelatin and $30 \%$ albumin, both in PBS, $\mathrm{pH} 6$ ) fixed with $0.6 \%$ glutaraldehyde, according to the protocol described in Levin (2004), and then sectioned at $50 \mu \mathrm{m}$ thickness using a Leica VT1000S Vibratome. Whole mount antibody staining was performed using an anti-muscle ATPase 12/101 monoclonal antibody (Developmental Studies Hybridoma Bank) following the procedure described in Ori et al., (2006).

\section{Acknowledgments}

We thank Jean-Paul Saint-Jeannet for providing XSox9 CDNA. We are particularly grateful to Guglielma De Matienzo, Marzia Fabbri, Elena Landi and Salvatore Di Maria for technical assistance and frog care. The work was supported by grant from Ministero dell'Istruzione, dell'Università e della Ricerca (MIUR, Project: "Functional analysis of the hyaluronanhyaluronan receptor system during development and in genetic diseases").

\section{References}

BERNARD, P., GABANT, P., BAHASSI, E.M. and COUTURIER, M. (1994). Positive Selection Vectors Using the F Plasmid ccdB Killer Gene. Gene148: 7174.

BRADBURY, E.J., MOON, L.D., POPAT, R.J., KING, V.R., BENNETT, G.S., PATEL, P.N., FAWCETT, J.W. and MCMAHON, S.B. (2002). Chondroitinase $A B C$ promotes functional recovery after spinal cord injury. Nature 416: 636-640.

CAMENISCH, T.D., SPIDER, A.P., BREHM-GIBSON, T., BIESTERFELDT, J., AUGUSTINE, M., CALABRO, A., KUBALAK, S., KLEWER, S. and MCDONALD, J.A. (2000). Disruption of hyaluronan synthase-2 abrogates normal cardiac morphogenesis and hyaluronan-mediated transformation of epithelium to mesenchyme. J. Clin. Invest. 106: 349-360.

CATTARUZZA, S. and PERRIS, R. (2006). Approaching the proteoglycome: molecular interactions of proteoglycans and their functional output. Macromol Biosci. 6: 667-80.

COLLAZO, A., BRONNER-FRASER, M. and FRASER, S.E. (1993). Vital dye labelling of Xenopus laevis trunk neural crest reveals multipotency and novel pathways of migration. Development 118: 363-376.

DUTT, S., MATASCI, M., SOMMER, L. and ZIMMERMANN, D.R. (2006). Guidance of neural crest cell migration: the inhibitory function of the chondroitin sulfate proteoglycan, versican. Scientific World Journal. 6: 1114-1117.

FROHMAN, M.A. (1993). Rapid Amplification of Complementary DNA Ends for Generation of Full-Length Complementary DNAs: Thermal RACE. Methods in Enzymology 218: 340-356.

HARLAND, R.M. (1991). In situ hybridization: an improved whole-mount method for Xenopus embryos. Methods Cell Biol. 36: 685-695. 
IOZZO, R.V. (2005). Basement membrane proteoglycans: from cellar to ceiling. Nat. Rev. Mol. Cell Biol. 8: 646-656.

KROTOSKI, D.M., FRASER, S.E. and BRONNER-FRASER, M. (1988). Mapping of neural crest pathways in Xenopus laevis using inter- and intra-specific cell markers. Dev. Biol. 127: 119-132.

LANDOLT, R.M., VAUGHAN, L., WINTERHALTER, K.H. and ZIMMERMANN, D.R. (1995). Versican is selectively expressed in embryonic tissues that act as barriers to neural crest cell migration and axon outgrowth. Development 121: 2303-2312.

LEVIN, M. (2004). A novel immunohistochemical method for evaluation of antibody specificity and detection of labile targets in biological tissue. J. Biochem. Biophys. Methods. 58: 85-96.

MJAATVEDT, C.H.,'YAMAMURA, H., CAPEHART, A.A., TURNER, D. and MARKWALD, R.R. (1998). The Cspg2 gene, disrupted in the hdf mutant, is required for right cardiac chamber and endocardial cushion formation. Dev. Biol. 202: 56-66.

NARDINI, M., ORI, M., VIGETTI, D., GORNATI, R., NARDI, I. and PERRIS, R. (2004). Regulated gene expression of hyaluronan synthases during Xenopus laevis development. Gene Expr. Pattern. 43: 303-308.

NIEUWKOOP, P.D. and FABER, J. (1967). Normal table of Xenopus laevis (Daudin), a systematical and chronological survey of the development from the fertilized egg till the end of metamorphosis. Amsterdam: North-Holland Publishing Company. pp. 250.

ORI, M., NARDINI, M., CASINI, P., PERRIS, R. and NARDI, I. (2006). XHas2 activity is required during somitogenesis and precursor cell migration in Xenopus development. Development 133: 631-640.

PERISSINOTTO, D., IACOPETTI, P., BELLINA, I., DOLIANA, R., ‘COLOMBATTI, A., PETTWAY, Z., BRONNER-FRASER, M., SHINOMURA, T., KIMATA, K., MÖRGELIN, M., LÖFBERG, J. and PERRIS, R. (2000). Avian neural crest cell migration is diversely regulated by the two major hyaluronan-binding proteoglycans PG-M/versican and aggrecan. Development 127: 2823-2842.

PERRIS, R., PERISSINOTTO, D., PETTWAY, Z., BRONNER-FRASER, M., MORGELIN, M. and KIMATA, K. (1996). Inhibitory effects of PG-H/aggrecan and PG-M/versican on avian neural crest cell migration. FASEBJ. 10: 293-301.

PERRIS, R. (1997). The extracellular matrix in neural crest-cell migration. Trends Neurosci. 20: 23-31.

PERRIS R. and PERISSINOTTO D. (2000). Role of the extracellular matrix during neural crest cell migration. Mech Dev. 95: 3-21.

SCHMALFELDT, M., BANDTLOW, C.E., DOURS-ZIMMERMANN, M.T., WINTERHALTER, K.H. and ZIMMERMANN, D.R. (2000). Brain derived versican V2 is a potent inhibitor of axonal growth. J. Cell. Sci. 113: 807-816.

SHINOMURA, T., NISHIDA, Y., ITO, K. and KIMATA, K. (1993). cDNA cloning of $P G-M$, a large chondroitin sulfate proteoglycan expressed during chondrogenesis in chick limb buds. Alternative spliced multiforms of PG-M and their relationships to versican. J. Biol. Chem. 268: 14461-14469.

SPOKONY, R.F., AOKI, Y., SAINT-GERMAIN, N., MAGNER-FINK, E. and SAINTJEANNET, J.P. (2002). The transcription factor Sox9 is required for cranial neural crest development in Xenopus. Development 129: 421-432.

STIGSON, M., LÖFBERG, J. and KJELLÉN, L. (1997). Reduced epidermal expression of a PG-M/versican-like proteoglycan in embryos of the white mutant axolotl.Exp. Cell Res. 236:57-65.

STRAHLE, U., BLADER, P., ADAM, J. and INGHAM, P.W. (1994). A simple and efficient procedure for non-isotopic in situ hybridization to sectioned material. Trends Genet. 10: 75-76.

WU, Y.J., LA PIERRE, D.P., WU, J., YEE, A.J. and YANG, B.B. (2005). The interaction of versican with its binding partners. Cell Res. 15: 483-494.

ZIMMERMANN, D.R. and RUOSLAHTI, E. (1989). Multiple domains of the large fibroblast proteoglycan, versican. EMBO J. 8: 2975-2981. 


\section{Further Related Reading, published previously in the Int. J. Dev. Biol.}

See our recent Special Issue Developmental Biology in Poland edited by Tarkowski, Maleszewski and Kloc at: http://www.ijdb.ehu.es/web/contents.php?vol=52\&issue=2-3

See our recent Special Issue Ear Development edited by Fernando Giraldez and Bernd Fritzsch at: http://www.ijdb.ehu.es/web/contents.php?vol=51\&issue=6-7

$\mathrm{N}$-acetylgalactosamine 4-sulfate 6-O-sulfotransferase expression during early mouse embryonic development Ana-Marisa Salgueiro, Mário Filipe and José-António Belo

Int. J. Dev. Biol. (2006) 50: 705-708

Msx1 and Msx2 have shared essential functions in neural crest but may be dispensable in epidermis and axis formation in Xenopus

Deepak Khadka, Ting Luo and Thomas D. Sargent

Int. J. Dev. Biol. (2006) 50: 499-502

Glycosaminoglycans in early chick embryo.

Spyros S Skandalis, Achilleas D Theocharis, Nikoletta Papageorgakopoulou and Nikolas Zagris

Int. J. Dev. Biol. (2003) 47: 311-314

Palatal shelf adhesion is mediated by TGF-beta(3) induced chondroitin sulphate proteoglycan

A Gato, C Tudela, I Alonso, JA Moro, MJ Rivas, MA Formoso, C Maestro, A Del Rio, J Murillo, C Barrio, C Martinez-Alvarez Int. J. Dev. Biol. (2001) 45: S97-S98

Local increase level of chondroitin sulfate induces changes in the rhombencephalic neural crest migration.

J A Moro Balbás, A Gato, M Alonso and E Barbosa

Int. J. Dev. Biol. (1998) 42: 207-216

Divide, accumulate, differentiate: cell condensation in skeletal development revisited.

B K Hall and T Miyake

Int. J. Dev. Biol. (1995) 39: 881-893

Collective cell migration in morphogenesis and cancer

Peter Friedl, Yael Hegerfeldt and Miriam Tusch

Int. J. Dev. Biol. (2004) 48: 441-449

Extracellular matrix and its receptors during development.

D Gullberg and P Ekblom

Int. J. Dev. Biol. (1995) 39: 845-854

Origins and plasticity of neural crest cells and their roles in jaw and craniofacial evolution.

Paul A Trainor, Kristin R Melton and Miguel Manzanares

Int. J. Dev. Biol. (2003) 47: 541-553

Regulation of neural crest cell populations: occurrence, distribution and underlying mechanisms.

$\mathrm{J} L$ Vaglia and B K Hall

Int. J. Dev. Biol. (1999) 43: 95-110
2006 ISI ${ }^{* *}$ Impact Factor $=3.577^{* *}$
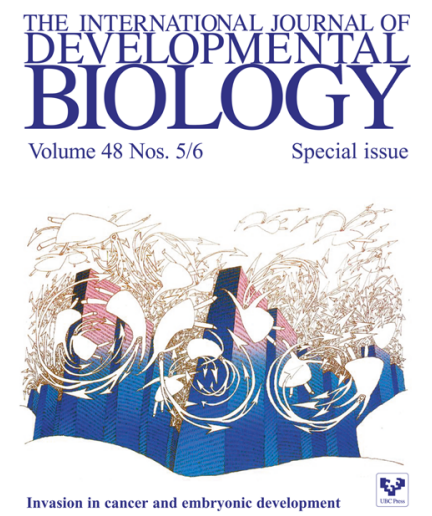